Cellular growth and proliferation, Generation of cells

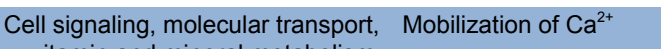
vitamin and mineral metabolism

$\begin{gathered}\text { Nervous system development and } \\ \text { function, tissue morphology }\end{gathered}$
$\begin{gathered}\text { Nervous system development and neurons } \\ \text { function, tissue morphology }\end{gathered}$
$\begin{gathered}\text { Quantity of nervous } \\ \text { tissue }\end{gathered}$

Behavior Learning

$\begin{array}{lc}\text { Behavior } & \text { Cognition } \\ \begin{array}{l}\text { Cellular development, cellular } \\ \text { growth and proliferation, } \\ \text { hematological system } \\ \text { development and function, } \\ \text { hematopoiesis, lymphoid tissue } \\ \text { structure and development, } \\ \text { tissue development }\end{array} & \begin{array}{c}\text { Development of } \\ \text { leukocytes }\end{array} \\ \begin{array}{l}\text { Cellular development, nervous } \\ \text { system development and } \\ \text { function, tissue development }\end{array} & \begin{array}{c}\text { Differentiation of } \\ \text { neurons }\end{array} \\ \begin{array}{l}\text { Nervous system development and } \\ \text { function }\end{array} & \begin{array}{c}\text { Sensory system } \\ \text { development }\end{array}\end{array}$

Cellular development, cellular growth and proliferation, hematological system development and function Cellular development

Cell proliferation of leukocyte cell lines nervous system

Cellular development, hematological system development and function, hematopoiesis, lymphoid tissue structure and development

Cellular development, hematological system development and function, hematopoiesis, lymphoid tissue structure and development

Cellular development, cellular growth and proliferation, endocrine system development and function, organ development

Proliferation of pancreatic cells
5.5 ABI2,ADGRB3,ALOX5,ASIC2,ASZ1,ATOH1,ATOH7,AVPR1A,BBC3,BTG2,CAMP CAPZA3,CBLN1,CD1D,CD40LG,CFAP54,CIITA,CNTN2,CR2,CRISP2,CSF3, CTNNA2,CUX2,CXCL11,DAZ2,DCC,DCX,DPYSL5,DSCAM,EBF3,EPHA3,EPHB1, ERG,FAS,FGF2,FGFR2,FLT1,FLT3,FSHB,GALNTL5, GDNF, GFAP,GNAO1,HAND1, HGF,HLA-DQA1,HNF1A,HOXA13,IFNL3,IGF2,IGHG1, IKZF1, IL1RL1,IL20RA,IL23A, IL2RA,KCNJ2,KDF1,KISS1R,LCK,LHX6,LRAT,LRRC7,LRRTM4,MAS1,MBP,MEI4, MSR1,MUC2,MYH11,MYO16,NCKAP1L,NKX2-8,NLGN3,NPPA,NRG1,NTRK3, OLFM3,OTX2,PARP1,PAX3,PAX5,PBX3,PCDHB6,PDILT,PGR,PIP5K1B,PLG, POU2AF1,PRDM14,PRLR,PROK1,PROK2,PTPRC,RBMY1A1 (includes others), RIMS1,RIPK3,RIT2,RPGRIP1,RTN3,RXRG,S100B,SHANK2,SIT1,SLAMF1, SLC17A6,SLC5A7,SLIT1,SLITRK3,SOX5,SPATA22,ST8SIA2,TFAP2A,TFAP4, THEMIS,TMEM106B, TRAT1,TSSK2,UBR2, VEGFD,VPREB1,VWC2,WFDC2,WNT2, WNT4,XRCC5,ZC3H13

4.2 ALOX12,AVPR1A,CAMP,CCL23,CCL8,CR2,CX3CR1,CXCL11,CXCL17,DIO2, FCRL5,GNAO1,HTR2A,HTR2C,IGHG1,KISS1R,LCK,MAS1,MLNR,PKHD1, PPP1R1B,PROK2,PTPRC,SRL,TPSD1,TRAT1,WNT4,XCL1

3.8 ATOH1,ATOH7,CNTN2,CUX2,CX3CR1,DCC,DSCAM,FGF2,FGFR2,GAD1,GDF15, GDNF,GNAO1,GNAS,GRID1,GRIN2B,HGF,KISS1R,LHX6,MDM2,NKX2-8,NLGN3, NRG1,NTRK3,OTX2,RIMS1,SLC17A6,ST8SIA2,TBR1,TFAP2A,VAX1,WNT4,XRCC5

3.6 ATOH1,ATOH7,CNTN2,CUX2,CX3CR1,DCC,DSCAM,FGF2,FGFR2,GAD1, GDF15, GDNF,GNAO1,GNAS,GRID1,GRIN2B,HGF,KISS1R,LHX6,MDM2,NKX2-8,NLGN3, NRG1,NTRK3,OTX1,OTX2,RIMS1,SLC17A6,ST8SIA2,TBR1,TFAP2A,VAX1,WNT4, XRCC5

3.5 ADAMTS20,ALOX12,ALOX5,ARL11,ASPN,ATOH1,AVPR1A,BHLHE22,BRINP1, BTG2,CAMP,CBLN1,CD1D,CD300LF,CD40LG,CDH23,CIITA,CNTN2,CR2,CSF3, CSRP3,CTNNA2,DCX,DISP3,DPYSL5,DSCAM,EBF3,ELAVL2,EPHB1,ERG,FABP2, FAS,FGF2,FGFR2,FLT1,FLT3,FOXD3,FSHB, GATA5, GDNF, GLP1R,GNAO1,GNAS, HAND1, HGF,HLA-DQA1,HNF1A,HTR2A,HTR2C, IFNA8,IFNK, IFNL3,IFNW1, IGF2, IGHG1,IGK,IKZF1, IKZF3,IL1RL1,IL20RA,IL23A,IL2RA,IRF6,KDF1,KRT84,LCK, LHX3,LHX6,LOR,LRRC7,MB,MDM2,MEOX1,mir-122,MYF6,MYH11,NCKAP1L,NFIX, NKX3-2,NLGN3,NMRK2,NR4A3,NRG1,NTRK3,OGN,OSTN,OTX1,OTX2,PARP1, PAX3,PAX5,PCSK2,PGR,PLG,POU2AF1,PRDM14,PRDM16,PRLR,PROK1,PROM1, PTPRC,RBM46,RIMS1,RIPK3,RIT2,S100B,SERPINB2,SFRP5,SHANK2,SHC4, SIGLEC10,SIT1,SLAMF1,SLIT1,SOX5,SPON1,ST8SIA2,TBL1X,TBR1,TBX1, TFAP2A,TFAP2B, TFAP4, THEMIS, THSD1,TRAT1,TRPM2,UBR2,USP28,VAX1, VEGFD, VPREB1,VWC2,WDR62,WNT16,WNT4,XRCC5

3.0 ABI2,ADGRB3,AVPR1A,BRINP1,BTG2,CNTN2,CSF3,CUX2,CX3CR1,EPHA6,GJB6, GLP1R,GRIN2A,GRIN2B,HRH3,HTR2A,HTR2C,IGF2,KCNJ5,LRRC7,NLGN3,NRG1, NTRK3,PAH,PAK5,PCDH8,PEX5L,PPP1R1B,RPS6KA5,S100B,SHANK2,ST8SIA2, TBR1,TBX1,UNC13C

2.9 ABI2,ADGRB3,AVPR1A,BRINP1,BTG2,CNGA4,CNTN2,CSF3,CUX2,CX3CR1, EPHA6,GJB6,GLP1R,GNAS, GRIN2A,GRIN2B,HRH3,HTR2A,HTR2C,IGF2,KCNJ2, KCNJ5,LRRC7,NLGN3,NRG1,NTRK3,PAH,PAK5,PCDH8,PEX5L,PPP1R1B, RPS6KA5,S100B,SHANK2,ST8SIA2,TBR1,TBX1,UNC13C

2.8 BBC3,CD1D,CD40LG,CIITA,CR2,CSF3,CXCL11,EPHA3,EPHB1,FAS,FGF2,FLT1, FLT3,HLA-DQA1,HNF1A,IFNL3,IGF2,IKZF1,IL1RL1,IL23A,IL2RA,LCK,MSR1, MYH11,NCKAP1L,NPPA,PAX5,PGR,PLG,POU2AF1,PTPRC,RIPK3,SIT1,SLAMF1, SOX5,TFAP4,THEMIS,TRAT1,VPREB1,WNT4,XRCC5

2.8 ATOH1,BHLHE22,BRINP1,BTG2,CBLN1,CDH23,CNTN2,DISP3,EBF3,ELAVL2, FGF2,FGFR2,GDNF,HGF,IGK,LHX3,NFIX,NTRK3,OTX2,PAX5,RIT2,ST8SIA2,TBR1, TBX1,TFAP2A, UBR2,VAX1,VEGFD,VWC2,WDR62

2.6 ABI2,ATOH1,ATOH7,CDHR1,CRYGB,CX3CR1,DSCAM,EBF3,EPHB1,FGF2,FGFR2,C

\section{Differentiation of}

2.6 CSF3,FGF2,FGFR2,FLT1,FLT3,HIP1,IKZF1,IL22RA2,IL23A,IL23R,LCK, LOC102724788/PRODH,MBP,NRG1,NTRK3,PRDM16,PRLR,PTPRC

2.5 ATOH1,BHLHE22,BRINP1,BTG2,CBLN1,CDH23,CNTN2,DISP3,EBF3,ELAVL2, FGF2,FGFR2,GDNF,HGF,IGK,KISS1R,LHX3,NFIX,NLGN3,NRG1,NTRK3,OTX2, PAX3,PAX5, RIT2,SOX5,ST8SIA2,TBR1,TBX1,TFAP2A, UBR2,VAX1, VEGFD, VWC2, WDR62,WNT4

2.5 ALOX5,AVPR1A,CAMP,CD1D,CD40LG,CIITA,CR2,CSF3,FAS,FLT3,HGF,HLA-DQA1, HNF1A,IFNA8,IFNK,IFNL3,IFNW1,IGF2,IKZF1,IKZF3,IL23A,IL2RA, LCK,MYH11, NCKAP1L,PARP1,PAX5,POU2AF1,PRDM16,PTPRC,RIPK3,SFRP5,SIGLEC10,SIT1, SLAMF1,SOX5,TFAP4,THEMIS,TRAT1,TRPM2,VPREB1,WNT4,XRCC5

2.4 AVPR1A,CAMP,CD1D,CD40LG,CIITA,CR2,CSF3,FAS,FLT3,HGF,HLA-DQA1, HNF1A,IFNA8,IFNK,IFNL3,IFNW1,IGF2,IKZF1,IKZF3,IL23A,IL2RA, LCK,MYH11, NCKAP1L,PAX5,POU2AF1,PTPRC,RIPK3,SFRP5,SIGLEC10,SIT1,SLAMF1,SOX5, TFAP4,THEMIS, TRAT1,VPREB1,WNT4,XRCC5
2.4 FGF2,FOXD3,GAD2,GNAS,HGF,HNF1A,NR4A3,PGR,TPSD1 


\begin{tabular}{|c|c|}
\hline Categories $^{a}$ & $\begin{array}{l}\text { Diseases or functions } \\
\text { annotation }\end{array}$ \\
\hline Cellular growth and proliferation & $\begin{array}{l}\text { Cell proliferation of } \\
\text { hematopoietic cell } \\
\text { lines }\end{array}$ \\
\hline $\begin{array}{l}\text { Cellular growth and proliferation, } \\
\text { lymphoid tissue structure and } \\
\text { development }\end{array}$ & $\begin{array}{l}\text { Proliferation of } \\
\text { lymphatic system } \\
\text { cells }\end{array}$ \\
\hline $\begin{array}{l}\text { Cell death and survival, cellular } \\
\text { compromise }\end{array}$ & $\begin{array}{l}\text { Cytotoxicity of } \\
\text { cytotoxic T cells }\end{array}$ \\
\hline Behavior & Conditioning \\
\hline Cellular movement & Mobility of cells \\
\hline $\begin{array}{l}\text { Behavior, nervous system } \\
\text { development and function }\end{array}$ & Memory \\
\hline $\begin{array}{l}\text { Cellular development, connective } \\
\text { tissue development and function, } \\
\text { tissue development }\end{array}$ & $\begin{array}{l}\text { Differentiation of } \\
\text { stromal cells }\end{array}$ \\
\hline $\begin{array}{l}\text { Embryonic development, } \\
\text { organismal development, tissue } \\
\text { development }\end{array}$ & $\begin{array}{l}\text { Expansion of } \\
\text { embryonic tissue }\end{array}$ \\
\hline $\begin{array}{l}\text { Cellular development, cellular } \\
\text { growth and proliferation, } \\
\text { endocrine system development } \\
\text { and function }\end{array}$ & $\begin{array}{l}\text { Proliferation of apud } \\
\text { cells }\end{array}$ \\
\hline $\begin{array}{l}\text { Auditory and vestibular system } \\
\text { development and function, } \\
\text { nervous system development } \\
\text { and function }\end{array}$ & Hearing \\
\hline $\begin{array}{l}\text { Gastrointestinal disease, } \\
\text { organismal injury and } \\
\text { abnormalities }\end{array}$ & Diarrhea \\
\hline Cell death and survival & $\begin{array}{l}\text { Apoptosis of granulosa } \\
\text { cells }\end{array}$ \\
\hline $\begin{array}{l}\text { Developmental disorder, } \\
\text { immunological disease, } \\
\text { organismal injury and } \\
\text { abnormalities }\end{array}$ & $\begin{array}{l}\text { Hypoplasia of lymph } \\
\text { node }\end{array}$ \\
\hline $\begin{array}{l}\text { Gastrointestinal disease, } \\
\text { inflammatory response, } \\
\text { organismal injury and } \\
\text { abnormalities }\end{array}$ & $\begin{array}{l}\text { Inflammation of } \\
\text { intestine }\end{array}$ \\
\hline $\begin{array}{l}\text { Organismal injury and } \\
\text { abnormalities, renal and } \\
\text { urological disease }\end{array}$ & Failure of kidney \\
\hline $\begin{array}{l}\text { Organismal injury and } \\
\text { abnormalities, renal and } \\
\text { urological disease }\end{array}$ & Glomerulosclerosis \\
\hline $\begin{array}{l}\text { Cellular compromise, ophthalmic } \\
\text { disease, organismal injury and } \\
\text { abnormalities, tissue morphology }\end{array}$ & $\begin{array}{l}\text { Degeneration of } \\
\text { photoreceptors }\end{array}$ \\
\hline $\begin{array}{l}\text { Neurological disease, organismal } \\
\text { injury and abnormalities }\end{array}$ & Seizure disorder \\
\hline
\end{tabular}

Gastrointestinal disease, inflammatory disease, inflammatory response, organismal injury and abnormalities

Infectious diseases Bacterial infections

Gastrointestinal disease, $\quad$ Gastroenteritis
inflammatory disease

Ophthalmic disease, organismal Retinal degeneration injury and abnormalities

Neurological disease, organismal Seizures injury and abnormalities

\begin{tabular}{cc} 
Organismal injury and & Urination disorder \\
abnormalities, renal and & \\
urological disease & \\
$\begin{array}{c}\text { Developmental disorder, } \\
\text { organismal injury and }\end{array}$ & $\begin{array}{c}\text { Hypoplasia of } \\
\text { lymphatic system }\end{array}$ \\
\hline
\end{tabular}

2.3 CSF3,FGF2,FGFR2,FLT1,FLT3,HIP1,IKZF1,IL22RA2,IL23A,IL23R,LCK, LOC102724788/PRODH,MBP,NRG1,NTRK3,PAX5,PRDM16,PRLR,PTPRC

2.3 ABI2,ACPP,BBC3,BTN1A1,CAMP,CD1D,CD40LG,CIITA,CLEC4D,CLEC4G,CR1L, CR2,CSF3,EPHB1,ERG,FAS,FCRL3,FGF2,FGL2,FLT3,GAD1,GAD2,HGF, HLA-DQA1,IFNL3,IGF2,IGHG1,IKZF1, IKZF3, IL1RL1,IL23A,IL23R,IL2RA, ITGAD, KLK13,KLRD1,LCK,MBP,MDM2,NCKAP1L,NPPA,PARP1,POU2AF1,PTPRC,RIPK3, RPS6KA5,SIGLEC1,SIGLEC10,SIT1,SLAMF1,THEMIS,TP53INP1,TYR,UBASH3A VPREB1,VWF,XCL1,XRCC5

2.2 CD1D,CD40LG,IL23A,PTPRC,XCL1

2.2 ABI2,CX3CR1,EPHA6,GAD2,GDNF,GJD2,GLP1R,GPR26,GRIK2,GRIN2A,NLGN3, NLRP6,PPP1R1B,RPS6KA5,SLC17A6,ST8SIA2,TBR1

2.2 CGA,CSF3,FSHB, GAPDHS,NRG1

2.2 AVPR1A,BRINP1,CSF3,CUX2,CX3CR1,GJB6,GLP1R,GRIN2A,GRIN2B,HRH3, HTR2A,IGF2,KCNJ5,LRRC7,NRG1,NTRK3,PAK5,PCDH8,S100B,SHANK2,TBX1

2.2 CD40LG,FGF2,GDNF,PARP1,WNT4

\subsection{FGF2,HNF1A,MYF6,NRG1,REV3L}

\subsection{FGF2,FOXD3,GAD2,GNAS,HGF,HNF1A,NR4A3,PGR}

2.0 ASIC2,CDH23,CLIC5,FOXI1,GJB6,GRID1,GRXCR2,KCNA1,MBP,OTOR,PAX3, TBL1X,TBX1,TECTB, TFAP2A

-2.0 ALOX12,ALOX5,CYP8B1,DEFA6,FGF2,GUCY2C,HTR3D,IL2RA,PTPRC,SSTR5

-2.2 BTG2,CGA,FAS,FGF2,HGF

-2.2 CCRL2,CD40LG,SIT1,TRAT1,XRCC5

-2.3 ALOX12,ALOX5, CD40LG CIITA CR1L,CR2, CYP11B1, F10,FAS,FGFR2, GABRA6, GABRG3,GABRQ, GABRR2, GRIN2A, GRIN2B, GUCY2C,HGF,HLA-DQA1,IGF2, IL1RL1,IL22RA2,IL23A,IL23R,IL2RA,MUC2,NLRP6,PARP1,PGLYRP4,PLG,PTPRC, TP53INP1

-2.4 CD1D,CR1L,CSF3,F10,FCGR1B,FLT1,GABRA6,GABRG3,GDNF,HGF,IGHG1,IL2RA, MAS1,NPPA,RRM2B,SSTR5,VKORC1,WFDC2,WNT4

-2.4 CD1D,CR1L,CX3CR1,GAD2,GATA5,GDNF,HGF,IGHG1,IL2RA,MAS1,RRM2B, WFDC2

-2.4 CX3CR1,FGF2,GUCY2D,GUCY2F,IGF2,LRAT,OTX2,PROM1,RPGRIP1

-2.6 BBC3,CACNG4,CNTN2,DCX,FLT1,GABRA6,GABRG3,GABRQ,GABRR2,GAD1, GAD2,GDNF, GFAP,GNAO1,GRIK2, GRIK3, GRIN2A, GRIN2B,HTR1E,HTR2A,HTR2C, HTR3D,KCNA1,KCNA4,KCNC1,KCNK1,NR4A3,NRG1,OTX1,OTX2,PGR,PPP1R1B, PTPRC,RIMS1,SCN2B,SLC13A1,SLITRK3

-2.6 ALOX12,ALOX5,CD40LG,CIITA,CR1L,CR2,CYP11B1,F10,FAS,FGFR2,GABRA6, GABRG3,GABRQ, GABRR2, GRIN2A, GRIN2B, GUCY2C,HGF,HLA-DQA1,IL1RL1, IL22RA2,IL23A,IL23R,IL2RA,MUC2,NLRP6,PGLYRP4,PLG,PTPRC,TP53INP1

-2.7 CAMP,CCL8,CCRL2,CD1D,CLEC4D,CR2,CSF3,FCGR1B,FGFR2,GABRA6, GABRG3, GDF15, GRIN2A, GRIN2B, GUCY2C,IGK,IL23A,IL23R,IL2RA,ITGAD, LILRB5,MBL2,MSR1,NRG1,PARP1,PLG,RIPK3,RPS6KA5,SIGLEC1,SLAMF1, TLR10,TRPM2,VWF

-2.7 ALOX12,ALOX5,CD40LG,CIITA,CR1L,CR2,CYP11B1,F10,FAS,FGFR2,GABRA6, GABRG3,GABRQ,GABRR2,GRIN2A,GRIN2B, GUCY2C,HGF,HLA-DQA1,HTR3D, IL1RL1,IL22RA2,IL23A,IL23R,IL2RA,MUC2,NLRP6,PGLYRP4,PLG,PTPRC, TP53INP1,XCL1

-2.7 ASIC2,CDH23,CDHR1,CIITA,CX3CR1,FGF2, GUCY2D,GUCY2F,HTR2A,IGF2,LRAT, NTRK3,OTX2,PROM1,RDH12,RDH8,RIMS1,RPGRIP1

-2.8 BBC3,CACNG4,CNTN2, GABRA6,GABRG3,GABRQ, GABRR2, GAD1, GAD2,GDNF, GFAP,GNAO1,GRIK2, GRIK3,GRIN2A, GRIN2B,HTR2C,KCNA1,KCNA4, KCNC1,KCNK1,NR4A3,NRG1,OTX1,PGR,PTPRC,RIMS1,SCN2B,SLC13A1, SLITRK3

-3.0 ASIC2,CD1D,CR1L,FAS,FCAR,FLT1,FXYD4,GATA5,GDF15,HGF,HNF1A,IKZF3, KCNK1,MAS1,MTRR,MYH11,NPPA,PGR,RRM2B,SLC13A1,VWF

-3.7 CCRL2,CD40LG,IKZF1,LCK,MDM2,POU2AF1,PTPRC,RPS6KA5,RRM2B,SIT1, TBX1,TRAT1,VEGFD,XRCC5 


\begin{tabular}{|c|c|c|c|}
\hline Categories $^{\mathrm{a}}$ & $\begin{array}{l}\text { Diseases or functions } \\
\text { annotation }\end{array}$ & z-score ${ }^{b}$ & Genes \\
\hline $\begin{array}{l}\text { Inflammatory response, organismal } \\
\text { injury and abnormalities }\end{array}$ & Inflammation of organ & & $\begin{array}{l}\text { ABCB11,ACE2,ALOX12,ALOX5,BTN1A1,CAMP,CCL23,CCL8,CD1D,CD300LF, } \\
\text { CD40LG,CIITA,CLEC4D,CLEC4G,CR1L,CR2,CSF3,CX3CR1,CXCL17,CYP11B1, } \\
\text { CYP3A5,CYP3A7,F10,FAS,FCGR1B,FGFR2,FLT1,FLT3,GABRA6,GABRG3,GABRQ, } \\
\text { GABRR2,GAD2,GATA5,GDNF,GFAP,GRIN2A,GRIN2B,GUCY2C,HGF,HLA-DQA1, } \\
\text { HLA-DRB5,HMGCS2,HRH3,HTR2A,HTR2C,IFNL3,IGF2,IGHG1,IKZF3,IL1RL1, } \\
\text { IL22RA2,IL23A,IL23R,IL2RA,KCNA1,KLK13,KLK6,KRT5,LCK,LOR,MBP,MDM2, } \\
\text { mir-122,MUC2,NLRP6,NRG1,PARP1,PGLYRP4,PGR,PLG,PNLIP,PROM1,PTPRC, } \\
\text { RDH8,RIPK3,RPS6KA5,RXRG,SERPINB3,SIGLEC10,SIT1,SOX5,TLR10,TP53INP1, } \\
\text { TYR,UBASH3A,VEGFD,VWF,WNT4,XCL1 }\end{array}$ \\
\hline
\end{tabular}

Information on the experimental condition is provided in the legends to S2 Fig.

${ }^{a}$ Analyese were conducted for 1265 genes that changed at $p<0.0073$. Blue area highlights annotations that also yielded the z-score of $>2$ or $<-2$ for 2234 genes whose expression changed at $p<0.0126$.

${ }^{\mathrm{b}}$ Annotations with the $\mathrm{z}$-score of $>2$ (indicative of activation) or $<-2$ (indicative of inhibition) are listed 